\title{
Dynamics in the filament of september 17 2010 and in its channel
}

\author{
Nicole Mein ${ }^{1}$, Pierre Mein ${ }^{2}$, Brigitte Schmieder ${ }^{2}$, Jean-Marie \\ Malherbe $^{2}$ and Thierry Roudier ${ }^{3}$ \\ ${ }^{1}$ Observatoire de Paris, Meudon, 92195, France \\ ${ }^{2}$ Observatoire de Paris, LESIA, Meudon, 92195, France \\ ${ }^{3}$ Observatoire Midi-Pyrénées, Toulouse, France
}

\begin{abstract}
Dynamics of a filament is investigated in $\mathrm{H} \alpha$. Counterstreaming flows are observed along the filament. Photospheric horizontal motions have been computed by using a Coherent Structure Tracking algorithm in the filament environment.
\end{abstract}

Keywords. Sun: prominences, granulation, velocity field

\section{Multi-wavelength observations}

We investigate the dynamics of a large filament in the central part of the active region NOAA 11106 observed near the central meridian on Sept 17 2010. This filament was observed in $\mathrm{H} \alpha$ with the THEMIS telescope in Tenerife in both modes (MTR and MSDP) and in $304 \AA$ with the EUV imager (AIA: Lemen et al. 2012) on board of the Solar Dynamics Observatory (SDO).

Figures (1) and (2) show two sets of $\mathrm{H} \alpha$ intensity and dopplershift images obtained with the Multichannel Subtractive Double Pass (MSDP) spectrograph operating on the THEMIS telescope (Tenerife). The velocities are calculated by the bisector method. If we assume that the flows are horizontal, and taking into account the position on the disk, velocities may be multiplied by a factor 2 . An other possibility is to suppose that the filament is like a cloud over the chromosphere. In that case, the velocity may still be multiplied by an additional factor 2 . If we apply both corrections, the velocities could be estimated to be between $-10 \mathrm{~km} / \mathrm{s}$ and $20 \mathrm{~km} / \mathrm{s}$. The most striking feature is the upward velocity in the filament itself and the presence of downward velocities in the filament channel showing perhaps the signature of the counterstreaming flow detected also in $304 \AA$ movies. But the order of magnitude, between 50 to $150 \mathrm{~km} / \mathrm{s}$, is very different. Perhaps this is due to the filament direction or to the mixing of many threads in $\mathrm{H} \alpha$ observations along the line of sight. Figure (3a) shows the environment of the filament seen by AIA and Figure (3b) the magnetic field given by SDO/HMI. We can see that the filament lies between positive and negative polarities, as usual.

\section{Supergranule velocities computed with the CST algorithm}

The photospheric velocity flow has been calculated by the Coherent Structure Tracking algorithm (CST) (Roudier et al. 2009).

The cork distribution map in the filament environment has been derived by using the photospheric flow averaged over three hours. The corks are gathered at the edge of supergranules in the quiet Sun. In the facular regions, they are located on the brightness maxima in $1600 \AA$ (Figure 3c). The mean proper motion vectors over 3 hours calculated by CST are shown in Figure (3d) together with the contour of the filament and its channel (full lines). The field of view covers the two different fields of view of the MSDP. Two important features can be noticed: converging zones associated with barbs (one of them 

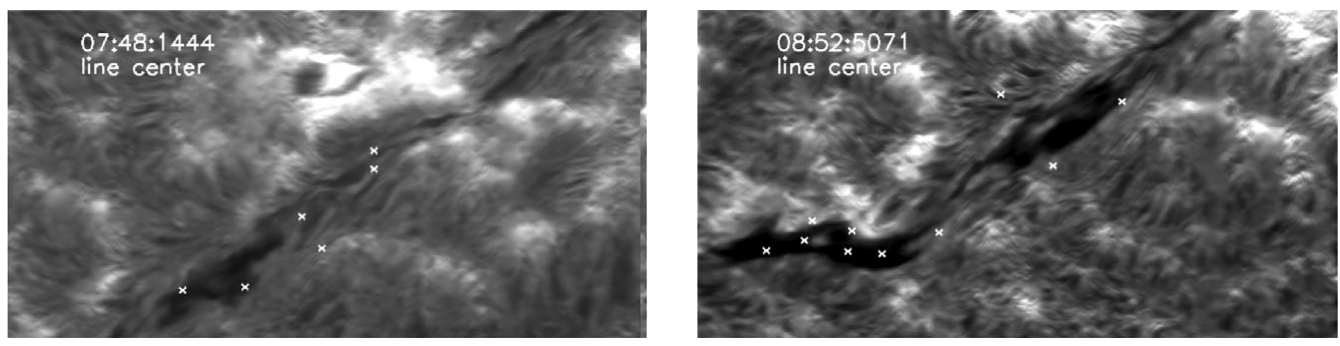

Figure 1. Intensities measured with MSDP at THEMIS
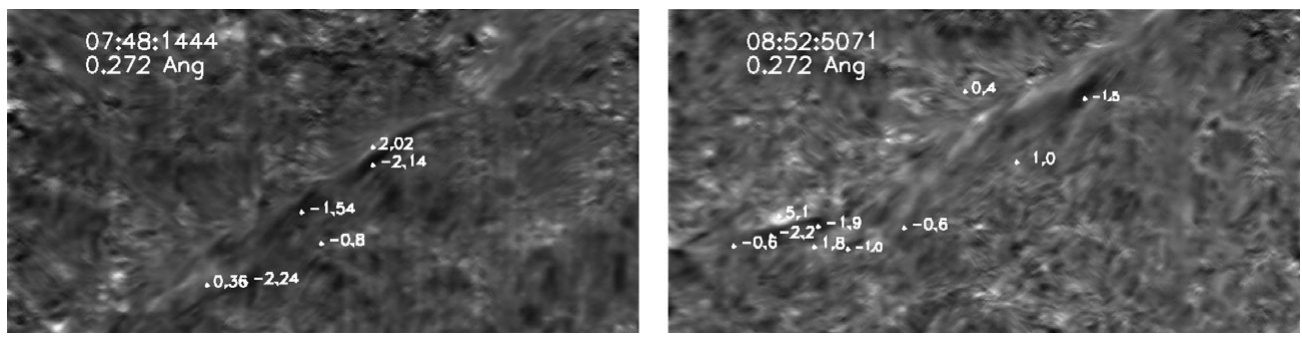

Figure 2. Dopplershifts $(\mathrm{km} / \mathrm{s})$ measured with MSDP at THEMIS

(a)
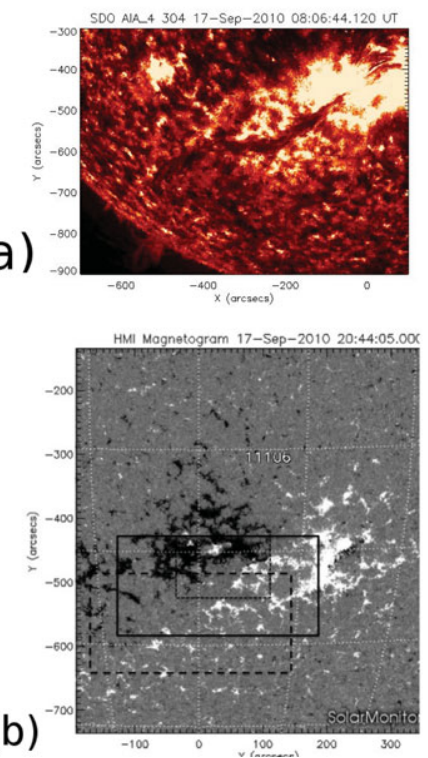
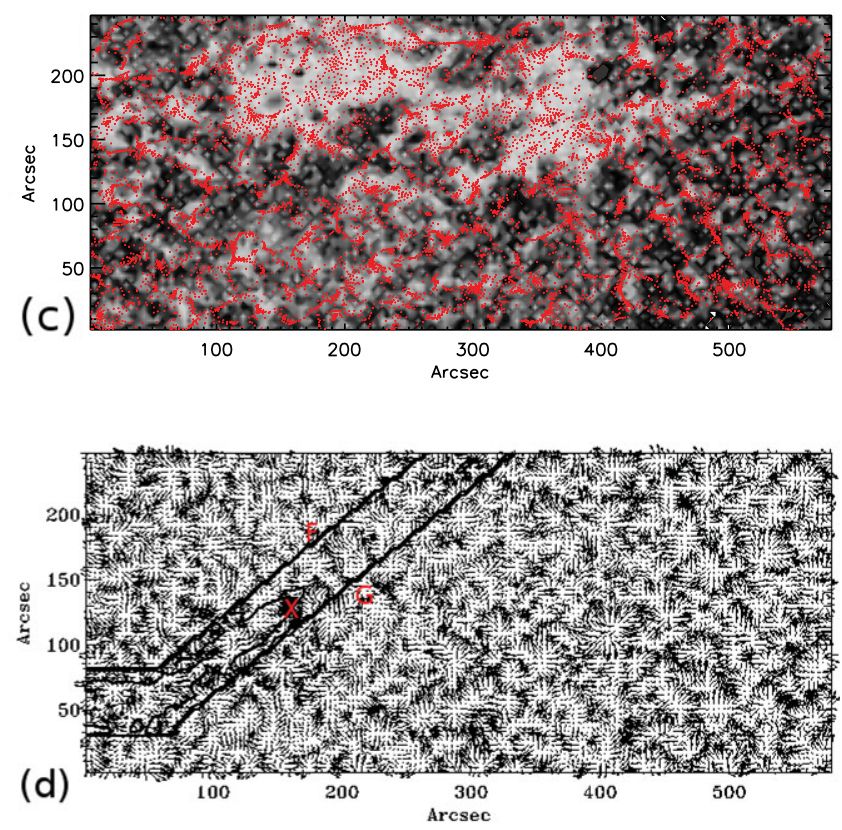

Figure 3. SDO AIA $304 \AA$ (a), HMI magnetic field (b), cork distribution map overlying an AIA image at $1600 \AA$ (c) and proper motion velocity vectors (d)

is indicated by a red/dark star), and a flow crossing the filament in its middle part ( $\mathrm{x}=$ 200 arc sec, $\mathrm{y}=150$ arc sec).

\section{References}

Roudier, Th., Rieutord, M., Brito, D., Rincon, F., Malherbe, J. M., Meunier, N., Berger, T., \& Frank, Z., 2009, A \& A], 496, 945

Lemen, J. R., Title, A. M., Akin, D. J., et al. 2012, Solar Phys. 275, 17 